\title{
Técnicas de avaliação dos valores energéticos e dos coeficientes de digestibilidade de alguns alimentos para emas (Rhea americana) em crescimento ${ }^{1}$
}

\author{
Reinaldo Lopes Morata2 ${ }^{2}$, Théa Mirian Medeiros Machado ${ }^{3}$, Luiz Fernando Teixeira Albino ${ }^{3}$, \\ Horacio Santiago Rostagno ${ }^{3}$, Edenio Detmann ${ }^{3}$, Laura Teodoro de Oliveira Fernandes ${ }^{4}$, \\ Henrique Nunes Parente ${ }^{2}$, Karine Vieira Antunes ${ }^{2}$, Aline Conceição Almeida ${ }^{5}$, Antônio Carlos \\ Csermak Júnior6
}

\footnotetext{
${ }^{1}$ Parte da tese de Mestrado do primeiro autor apresentada à Universidade Federal de Viçosa.

2 Mestrando em Zootecnia - Universidade Federal de Viçosa.

${ }^{3}$ Departamento de Zootecnia - Universidade Federal de Viçosa.

${ }^{4}$ Médica Veterinária - CDA - CBMM, Araxá, MG

${ }^{5}$ Doutoranda em Zootecnia - Universidade Federal de Viçosa.

${ }^{6}$ Graduação em Zootecnia - Universidade Federal de Viçosa.
}

RESUMO - Avaliaram-se os valores de energia metabolizável aparente e aparente corrigida (EMA e EMAn, respectivamente), os coeficientes de metabolização da energia bruta (CMEB e CMEBn) e da digestibilidade da MS e MO (CDAMS e CDAMO) de alguns alimentos para emas (Rhea americana) de sete meses de idade, utilizando-se as técnicas de coleta total de excretas e do óxido de cromo como indicador. Utilizou-se o delineamento experimental inteiramente ao acaso, em esquema de parcelas subdivididas, com seis tratamentos, cada um com três repetições. O experimento foi realizado três vezes, seqüencialmente. Os valores dos CDAMS, CDAMO, EMA, EMAn, CMEB e CMEBn dos alimentos, determinados por meio da técnica de coleta total de excretas, foram, de modo geral, superiores aos determinados pela técnica do óxido de cromo. A técnica do óxido de cromo mostrou-se inadequada para determinar os CDAMS, CDAMO, EMA, EMAn, CMEB e CMEBn dos alimentos para emas em comparação à técnica de coleta total de excretas. Recomenda-se a realização de ensaios similares envolvendo outras técnicas indiretas para estimar parâmetros de digestibilidade nesta espécie.

Palavras-chave: animais silvestres, coleta total, energia metabolizável, nutrição, óxido de cromo, Rhea americana

\section{Techniques of evaluation of the energy values and the coefficients of digestibility of some feedstuffs for growing greater rhea (Rhea Americana)}

\footnotetext{
ABSTRACT - It was evaluated the values of apparent metabolizable and apparent corrected energy (AME and AMEn, respectively), the coefficients of metabolization of gross energy (MCEC and MCECn) and the digestibility of DM and OM (ADDMC and ADOMC) of some feedstuffs for greater rhea averaging seven months old, by the techniques of total feces collection and chromium oxide as external marker. The experiment was analyzed as a complete randomized design with six treatments and three replications per experiment. The experiment was carried out three times senquentially. The values of ADDMC, ADOMC, AME, AMEn, MCEC and MCECn of feedstuffs determined by total feces collection were greater than those determined with chromium oxide. The use of marker was not efficient to determine ADDMC, ADOMC, AME, AMEn, MCEC and MCECn of the feedstuffs for greater rhea when compared to total feces collection method. It is recommended more studies of indirect techniques to estimate the digestibility parameters in similar assays.
}

Key Words: chromium oxide, metabolizable energy, nutrition, Rhea Americana, total collection, wild animal

\section{Introdução}

Apesar da riqueza de espécies da fauna silvestre nativa, o Brasil sempre subutilizou sua diversidade biológica, embora tenha se destacado nas últimas décadas como um dos principais exportadores de alimentos. A maior parte dos sistemas produtivos do agronegócio do país baseia-se em espécies exóticas.

Face às políticas e aos instrumentos governamentais de conservação da biodiversidade, surgiu a exploração racional e sustentável de espécies de animais silvestres com potencial zootécnico, entre as quais se destacam as 
criações de capivara (Hydrochaeris hydrochaeris), cateto (Tayassu tajacu), queixada (Tayassu pecari), paca (Agouti paca), jacaré-do-papo-amarelo (Cayman latirostris), jacarédo-pantanal (Caimam crocodilus yacare), pássaros ornamentais e ema (Rhea americana).

De acordo com dados disponibilizados pelo Instituto Brasileiro do Meio Ambiente e dos Recursos Naturais Renováveis - IBAMA, é significativo o aumento no número de registros de criadouros comerciais de ema desde a promulgação das portarias 117 e $118 \mathrm{em} 1997$. Esse aumento pode ser explicado pela perspectiva de boa rentabilidade, em virtude do potencial zootécnico e da rusticidade e pelo valor comercial da carne, do couro, das plumas, da gordura e dos ovos desta espécie. A criação comercial de ema é uma alternativa de diversificação e/ou integração de sistemas produtivos dentro da propriedade, respeitando-se os conceitos de produção economicamente viável, ecologicamente sustentável e socialmente justa (geração de empregos).

Para se obter sucesso na criação de emas, é importante que o criador conte com respaldo técnico-científico nas áreas de nutrição, reprodução, genética, sanidade, ambiência, etologia e manejo, denominados fatores produtivos, os quais fornecem subsídios para melhorar os desempenhos reprodutivo e produtivo dos sistemas de criação.

A formulação de dietas para emas baseia-se em conhecimentos empíricos e informações de pesquisas relacionadas a outras espécies. O seu desconhecimento pode ocasionar sub ou supernutrição dos animais, influenciando negativamente a eficiência produtiva e a rentabilidade do sistema.

O conhecimento da composição química dos alimentos, do seu conteúdo energético e da digestibilidade de cada nutriente possibilita aos nutricionistas formular dietas de custo mínimo para ótimo desempenho.

Considerando a escassez de informações sobre valores energéticos e de digestibilidade dos nutrientes nos alimentos utilizados em dietas para emas em crescimento, objetivou-se com este trabalho determinar e comparar os valores de EMA e EMAn e os coeficientes de metabolização da energia bruta e de digestibilidade aparente da MS e MO - obtidas pelas técnicas de coleta total de excretas e do óxido de cromo como indicador, simultaneamente - de três alimentos energéticos e dois protéicos comumente utilizados na indústria de ração animal.

\section{Material e Métodos}

Foram utilizadas 36 emas em fase de crescimento, em delineamento experimental inteiramente ao acaso, com seis tratamentos (cinco dietas experimentais e uma dieta referência), cada um com três repetições. O experimento foi conduzido em esquema de parcelas subdivididas, com os alimentos alocados às parcelas e as técnicas para estimação da excreção fecal (coleta total de excretas e uso do $\mathrm{Cr}_{2} \mathrm{O}_{3}$ como indicador) às subparcelas. Cada tratamento foi repetido três vezes, em intervalos de nove dias.

Os tratamentos consistiram de cinco alimentos e uma ração-referência. Os alimentos energéticos (milho, sorgo e farelo de trigo) substituíram $40 \%$ e os protéicos (farelo de soja e farinha de carne e ossos), $30 \%$ da ração-referência, segundo metodologia de substituição proposta por Matterson et al. (1965).

A ração-referência (Tabela 1) foi elaborada segundo as necessidades nutricionais adotadas por Saracura (1993), Sanchez et al. (1998) e Silva (2001). As composições químicas dos alimentos utilizados foram calculadas conforme recomendações de Rostagno et al. (2000).

Tabela 1 - Composições centesimal (matéria natural) e nutricional (matéria seca) da ração-referência

Table 1 - Ingredient (as-fed) and calculated (dry matter) compositions of the reference diet

\begin{tabular}{|c|c|}
\hline $\begin{array}{l}\text { Ingrediente } \\
\text { Ingredient }\end{array}$ & $(\%)$ \\
\hline Milho (Corn) & 64,0 \\
\hline Farelo de soja (Soybean meal) & 27,40 \\
\hline Óleo vegetal (Soybean oil) & 1,000 \\
\hline Fosfato bicálcico (Dicalcium phosphate) & 3,600 \\
\hline Calcário (Limestone) & 2,500 \\
\hline Sal (Salt) & 0,450 \\
\hline Suplemento mineral (Mineral supplement) ${ }^{1}$ & 0,2 \\
\hline Suplemento vitamínico (Vitamin supplement) $)^{2}$ & 0,250 \\
\hline Antioxidante ${\text { (Antioxidant })^{3}}^{3}$ & 0,01 \\
\hline Óxido de cromo $\left(\mathrm{Cr}_{2} \mathrm{O}_{3}\right)$ (Chromic oxide) & 0,500 \\
\hline Total & 100,00 \\
\hline
\end{tabular}

Valor calculado

Calculated value

MS $(D M)(\%)$

87,20

PB $(C P)(\%)$

18,45

MO $(O M)(\%)$

91,14

$\mathrm{EE}(\%)$

FDN $(N D F)(\%)$

3,56

FDA $(A D F)(\%)$

17,93

EB (GE) $(\mathrm{kcal} / \mathrm{kg})$

4,13

EM $(M E)(\mathrm{kcal} / \mathrm{kg})$

4.496

$\mathrm{Ca}(\%)$

3.326

1,96

$\mathrm{P}(\%)$

0,98

1 Suplemento mineral contendo por $\mathrm{kg}$ de produto (Mineral supplement per $\mathrm{kg}$ of product): $\mathrm{Fe}-100,0 \mathrm{~g}$; Co - 2,0 g; Cu - 20,0 g; Mg - 160,0 g; Zn - 100,0 g

I - 2,0 g; e Excipiente q.s.p. (inert) - $500 \mathrm{~g}$;

2 Suplemento vitamínico contendo por $\mathrm{kg}$ do produto (Vitamin supplement per $\mathrm{kg}$ of product): vit. A - 10.000 .000 U.I.; vit. D3 - 2.000.000 U.I.; vit. E - 30.000 U.I.; vit. B1 - 2,0 g; vit. B2 - 6,0 g; vit. B6 - 4,0 g; vit. B12 - 0,015 g; ác. pantotênico (pantothenic acid) - 12,0 g; biotina (biotin) - $0,1 \mathrm{~g}$; vit. K3 - 3,0 g; ác. fólico (folic acid) - 1,0 g; ác. nicotínico (nicotinic acid) - 50,0 g; Se - 250,0 mg; e Excipiente q.s.p. (inert) $-1.000 \mathrm{~g}$

${ }^{3}$ Butil hidroxi tolueno $99 \%$. 
Durante o período experimental, as emas receberam ração e água à vontade.

As 36 emas (18 machos e 18 fêmeas) foram doadas pelo Centro de Desenvolvimento Ambiental (CDA) da Companhia Brasileira de Metalurgia e Mineração (CBMM), localizada no município de Araxá, Minas Gerais.

As aves nasceram entre os meses de setembro e outubro de 2003 e apresentavam, respectivamente, machos e fêmeas, peso corporal de 4,3 e 4,7 kg e seis meses de idade, em média, no início do experimento. No início e final de cada experimento, as emas foram identificadas, pesadas e, posteriormente, distribuídas aleatoriamente entre os tratamentos.

O ensaio de campo teve duração de 38 dias (29/03 a 05/05/2004), divididos em três experimentos. Os experimentos foram constituídos de cinco dias de adaptação às dietas, cinco de coleta de amostras de ração e excretas e quatro dias de transição entre as dietas. O terceiro experimento consistiu de cinco dias de adaptação às dietas e cinco de coleta.

Para evitar que as excretas fossem contaminadas por materiais estranhos, foi realizada toalete na região da cloaca dos animais.

As aves foram alojadas em um galpão (62 x10 m e pé-direito de 3,6 m) com piso de cimento, laterais teladas até o teto, cobertas por cortinas de plástico com sistema de roldanas para o seu manejo, cobertura de telhas de amianto com lanternim de $1,00 \times 0,30 \mathrm{~m}$. O galpão foi dividido em 20 baias, 18 experimentais $(10 \times 2,8 \mathrm{~m})$, equipadas com campânula, bebedouro e comedouro. Em cada baia (unidade experimental), foram alojados um macho e uma fêmea.

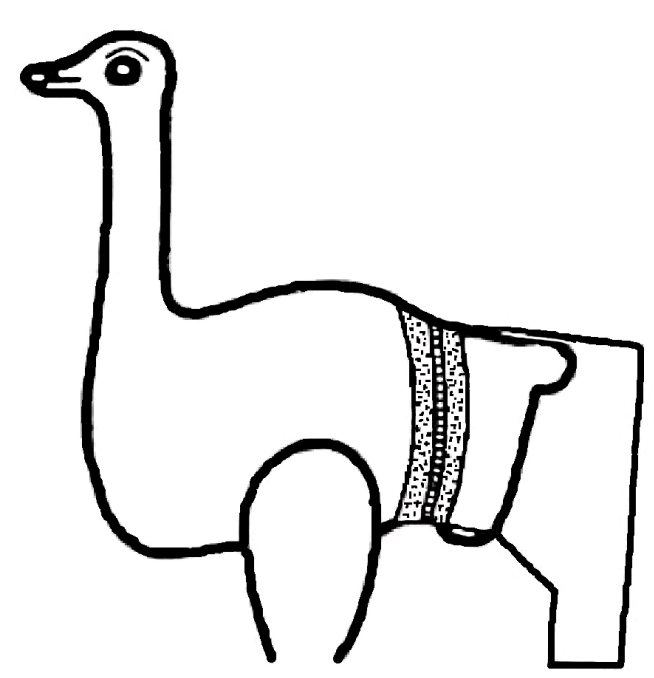

Figura 1 - Ilustração de um avestruz com bolsa coletora de excretas com cinto estomacal (Swart et al., 1993).

Figure 1 - Illustration of an ostrich with feces collection bag with stomachal belt (Swart et al., 1993).
Foram confeccionadas e utilizadas bolsas coletoras de plástico, adaptadas conforme metodologia de Swart et al. (1993) para avestruzes. No interior da bolsa coletora, foi acoplado saco plástico para facilitar a coleta total das excretas. À medida que os animais enchiam as bolsas com urina e/ou fezes, estas bolsas eram trocadas e os materiais eram pesados, identificados e armazenados sob refrigeração.

Ao término de cada experimento, foi determinada a quantidade de ração consumida por unidade experimental, durante os cinco dias de coleta em cada experimento.

Para a moagem, as excretas foram descongeladas e homogeneizadas por baia e por dia, efetuando-se a présecagem em estufas ventiladas a $65^{\circ} \mathrm{C}$. Alíquotas de $5 \mathrm{~g}$ de cada amostra/dia foram agrupadas para formar amostras compostas, as quais foram analisadas juntamente com os alimentos e as dietas, segundo metodologia descrita por Silva \& Queiroz (2002).

Uma vez obtidos os resultados das análises laboratoriais dos alimentos, das dietas experimentais e das excretas, foram calculados os valores de EMA e EMAn, utilizando-se as equações propostas por Matterson et al. (1965).

Aplicou-se a análise de variância conjunta de experimentos (Pimentel Gomes, 2000), comparando-se as médias pelo teste de Student-Newman-Keuls $(\alpha=0,05)$, por meio do SAS (1996).

\section{Resultados e Discussão}

Como a avaliação de alimentos para animais silvestres é uma linha de pesquisa relativamente recente e as informações sobre os valores energéticos e de digestibilidade dos nutrientes dos alimentos utilizados nas dietas para ratitas são escassos (não existem para emas), optou-se por comparar os valores estimados nesse trabalho aos determinados com avestruz, codornas e, especialmente, com frangos, pois as dietas para emas são formuladas com os valores estimados para frangos.

Os coeficientes de digestibilidade da matéria seca dos alimentos (milho, sorgo, farelo de trigo, farelo de soja e farinha de carne e ossos) obtidos pelas técnicas avaliadas encontram-se na Tabela 2.

Não houve efeito da interação técnica de coleta de excretas $\times$ alimento avaliado $(\mathrm{P}>0,05)$ para as variáveis CDAMS, CDAMO, EMAn, CMEB e CMEBn, porém, observou-se efeito $(\mathrm{P}<0,05)$ da interação técnica $\times$ alimento sobre a EMA.

Os coeficientes de digestibilidade aparente da matéria seca (CDAMS) dos alimentos diferiram estatisticamente $(\mathrm{P}<0,05)$ entre as metodologias adotadas. O valor médio obtido pela técnica de coleta total de excretas foi $11,42 \%$ superior em relação ao determinado com uso de indicador. 
Tabela 2 - Coeficientes de digestibilidade aparente da MS (CDAMS) dos alimentos, determinados pela técnica de coleta total de excretas e pelo uso de indicador

Table 2 - Values of the coefficients of apparent digestibility of DM (CADDM) of feedstuffs determined by the technique of total feces collection and use ofmarker

\begin{tabular}{|c|c|c|c|c|c|c|}
\hline \multirow[t]{2}{*}{$\begin{array}{l}\text { Técnica } \\
\text { Technique }\end{array}$} & \multicolumn{5}{|c|}{$\begin{array}{l}\text { CDAMS do alimento } \\
\text { ADDMC of feedstuffs }\end{array}$} & \multirow[t]{2}{*}{$\begin{array}{c}\text { Média }{ }^{1} \\
\text { Mean }\end{array}$} \\
\hline & $\begin{array}{l}\text { Milho } \\
\text { Corn }\end{array}$ & $\begin{array}{l}\text { Sorgo } \\
\text { Sorghum }\end{array}$ & $\begin{array}{l}\text { Farelo de trigo } \\
\text { Wheat meal }\end{array}$ & $\begin{array}{l}\text { Farelo de soja } \\
\text { Soybean meal }\end{array}$ & $\begin{array}{c}\text { Farinha de carne e ossos } \\
\text { Meat and bone meal }\end{array}$ & \\
\hline Total & 70,97 & 79,72 & 60,16 & 69,83 & 38,90 & $63,92 \mathrm{~A}$ \\
\hline $\begin{array}{l}\text { Indicador } \\
\text { Marker }\end{array}$ & 69,22 & 66,94 & 48,92 & 60,51 & 37,51 & $56,62 \mathrm{~B}$ \\
\hline Média $^{1}$ & $70,10 \mathrm{ab}$ & $73,33 \mathrm{a}$ & $54,54 \mathrm{c}$ & $65,17 \mathrm{~b}$ & $38,2 \mathrm{~d}$ & $\mathrm{CV}(\%)=22,12$ \\
\hline
\end{tabular}

${ }^{1}$ Médias seguidas por letras distintas são diferentes $(P<0,05)$ pelo teste SNK

1 Means followed by distinct letters differ $(P<0.05)$ by test SNK.

Ao comparar os CDAMS dos alimentos, observa-se que o sorgo apresentou o maior valor, porém, estatisticamente igual ao determinado para o milho e ambos maiores e estatisticamente diferentes do estimado para o farelo de trigo. Essa diferença pode ser explicada pela maior porcentagem de FDN e FDA no farelo de trigo, que geralmente apresenta baixa digestibilidade. A farinha de carne e ossos (FCO) resultou no menor CDAMS, o que poderia ser explicado pela maior quantidade de cinzas na farinha de carne e ossos.

O CDAMS determinado para o milho pela técnica de coleta total neste trabalho foi 17,$37 ; 24,90$ e $23,97 \%$ inferior aos valores encontrados por Albino et al. (1981), Coelho (1983) e Veloso et al. (1985) para frangos de corte e 16,51\% ao valor encontrado por Cilliers et al. (1997).

O CDAMS encontrado para o sorgo foi semelhante aos determinados por Albino et al.(1981)e Coelho (1983), porém foi $13,33 \%$ inferior ao apresentado por Veloso et al. (1985).

O CDAMS do farelo de trigo foi $33,74 \%$ superior aos valores encontrados na literatura para frangos de corte (Albino et al., 1981), 21,04\% (Veloso et al., 1985), 23,03\% (Borges et al., 2003).

Ao comparar os CDAMS determinados pela técnica de coleta total, observou-se que o valor encontrado para o farelo de soja foi 49,74; 45,00; 50,70 e 35,08\% superior aos encontrados, respectivamente, por Albino et al. (1981), Coelho (1983), Veloso et al. (1987) e Soares et al. (2005), em frango de corte.

O valor de CDAMS da farinha de carne e ossos foi $29,67 \%$ maior que os determinados por Albino et al. (1981) e Coelho (1983) e $42,05 \%$ superior ao determinado por Veloso et al. (1987).

Os coeficientes médios de digestibilidade aparente médio da matéria orgânica (CDAMO) dos alimentos foram diferentes estatisticamente $(\mathrm{P}<0,05)$ entre as metodologias adotadas (Tabela 3 ). O coeficiente médio obtido pela técnica de coleta total de excretas foi $8,54 \%$ superior ao determinado com uso de indicador.

O sorgo apresentou o maior CDAMO, porém o valor foi estatisticamente igual ao determinado para o milho e ambos maiores e estatisticamente diferentes do estimado para o farelo de trigo.

Os valores referentes à EMA e EMAn, expressos com base na matéria natural dos alimentos avaliados encontram-se, respectivamente, nas Tabelas 4 e 5 .

Houve diferença significativa $(\mathrm{P}<0,05)$ entre os valores médios de EMA do sorgo, farelo de trigo e farelo de soja obtidos pelas diferentes metodologias. As médias da EMA obtidas pela técnica de coleta total foram 13,07\% superiores para o sorgo, $16,05 \%$ para o farelo de trigo e $12,04 \%$ para o farelo de soja quando comparadas aos estimados com o uso de indicador. Entretanto, as médias da EMA do milho e da farinha de carne e ossos não diferiram $(\mathrm{P}>0,05)$ entre as técnicas utilizadas.

O valor de EMA do milho pela técnica de coleta total foi semelhante aos estimados com frangos por Albino et al. (1981), Rodrigues et al. (2001), Silva et al. (2003), D’Agostini et al. (2004) e Nagata et al. (2004), porém foi 6,22\% menor que os estimados por Coelho (1983), Veloso et al. (1985) e Albino et al. (1992), também com frangos e $8,43 \%$ que o determinado por Cilliers et al. (1997). Entretanto, foi $18,33 \%$ superior ao determinado por Torres (2003), em codornas. O valor de EMAn, no entanto, foi 2,92\% superior aos reportados por Rodrigues et al. (2001), 13,39\% maior que os estimados por Torres (2003), 4,83 e 7,96\% menor que os determinados em avestruzes (Cilliers et al., 1994, 1997, respectivamente), 4,39\% (em média) menor que os estimados com frangos (Coelho, 1983; Veloso etal., 1985; Embrapa, 1991; NRC, 1994; Rostagno et al., 2001) e $2,47 \%$ menor que os obtidos com codornas (Silva et al., 2003). Foi semelhante, porém, aos encontrados por Albino et al. (1992), D’Agostini et al. (2004), Blas et al. (2003) e Nagata et al. (2004), em frangos. 
Tabela 3 - Coeficientes de digestibilidade aparente da MO (CDAMO) dos alimentos, determinados pela técnica de coleta total de excretas e com o uso de indicador

Table 3 - Values of the coefficients of apparent digestibility of OM (CADOM) of feedstuffs determined by the technique of total feces collection and use of marker

\begin{tabular}{|c|c|c|c|c|c|c|}
\hline \multirow[t]{2}{*}{$\begin{array}{l}\text { Técnica } \\
\text { Technique }\end{array}$} & \multicolumn{5}{|c|}{$\begin{array}{l}\text { CDAMO do alimento } \\
\text { CADOM of feedstuff }\end{array}$} & \multirow[t]{2}{*}{$\begin{array}{c}\text { Média } \\
\text { Mean }\end{array}$} \\
\hline & $\begin{array}{l}\text { Milho } \\
\text { Corn }\end{array}$ & $\begin{array}{l}\text { Sorgo } \\
\text { Sorghum }\end{array}$ & $\begin{array}{l}\text { Farelo de trigo } \\
\text { Wheat meal }\end{array}$ & $\begin{array}{l}\text { Farelo de soja } \\
\text { Soybean meal }\end{array}$ & $\begin{array}{c}\text { Farinha de carne e ossos } \\
\text { Meat and bone meal }\end{array}$ & \\
\hline Total & 73,84 & 81,58 & 61,95 & 70,11 & 68,32 & $71,16 \mathrm{~A}$ \\
\hline $\begin{array}{l}\text { Indicador } \\
\text { Marker }\end{array}$ & 72,63 & 71,11 & 52,32 & 62,24 & 67,10 & $65,08 \mathrm{~B}$ \\
\hline Média ${ }^{1}$ & $73,24 a b$ & $76,34 \mathrm{a}$ & $57,13 \mathrm{c}$ & $66,17 \mathrm{~b}$ & $67,71 b$ & $\mathrm{CV}(\%)=15,09$ \\
\hline
\end{tabular}

${ }^{1}$ Médias seguidas por letras distintas são diferentes $(P<0,05)$ pelo teste SNK.

${ }^{1}$ Means followed of distinct letters differ $(P<0.05)$ by test SNK.

Tabela 4 - Valores de energia metabolizável aparente (EMA) dos alimentos, determinados pela técnica de coleta total de excretas e com uso de indicador

Table 4 - Values of apparent metabolizable energy (AME) of feedstuffs determined by the technique of total feces collection and use of marker

\begin{tabular}{lccccc}
\hline $\begin{array}{l}\text { Técnica } \\
\text { Technique }\end{array}$ & \multicolumn{5}{c}{$\begin{array}{c}\text { EMA do alimento } \\
\text { AME of feedstuff }\end{array}$} \\
\cline { 2 - 5 } & $\begin{array}{c}\text { Milho } \\
\text { Corn }\end{array}$ & $\begin{array}{c}\text { Sorgo } \\
\text { Sorghum }\end{array}$ & $\begin{array}{c}\text { Farelo de trigo } \\
\text { Wheat meal }\end{array}$ & $\begin{array}{c}\text { Farelo de soja } \\
\text { Soybean meal }\end{array}$ & $\begin{array}{c}\text { Farinha de carne e ossos } \\
\text { Meat and bone meal }\end{array}$ \\
\hline Total & $3.278,7 \mathrm{Aa}$ & $3.418,2 \mathrm{Aa}$ & $2.670,9 \mathrm{Ab}$ & $3.484,0 \mathrm{Aa}$ & $3.043,5 \mathrm{Aa}$ \\
$\begin{array}{l}\text { Indicador } \\
\text { Marker }\end{array}$ & $3.216,4 \mathrm{Aa}$ & $2.971,5 \mathrm{Ba}$ & $2.242,2 \mathrm{Bb}$ & $3.064,7 \mathrm{Ba}$ & $3.340,0 \mathrm{Aa}$
\end{tabular}

${ }^{1}$ Médias na mesma linha seguidas por letras minúsculas distintas ou na mesma coluna seguidas por letras maiúsculas distintas diferem $(\mathrm{P}<0,05)$ pelo teste SNK.

${ }^{1}$ Means in the same row followed by distinct small letters, or in the same column followed by distinct capital letters, differ $(P<0.05)$ by SNK test.

Tabela 5 - Valores de energia metabolizável aparente corrigida (EMAn), dos alimentos, determinados pela técnica de coleta total de excretas com uso de indicador

Table 5 - Values of corrected apparent metabolizable energy (AMEn), of feedstuffs determined by the technique of total feces collection and use of marker

\begin{tabular}{|c|c|c|c|c|c|c|}
\hline \multirow{2}{*}{$\begin{array}{l}\text { Técnica } \\
\text { Technique }\end{array}$} & \multicolumn{5}{|c|}{$\begin{array}{l}\text { EMAn do alimento }{ }^{1} \\
\text { AMEn of feedstuff }\end{array}$} & \multirow{2}{*}{$\begin{array}{l}\text { Média }^{1} \\
\text { Mean }\end{array}$} \\
\hline & $\begin{array}{l}\text { Milho } \\
\text { Corn }\end{array}$ & $\begin{array}{l}\text { Sorgo } \\
\text { Sorghum }\end{array}$ & $\begin{array}{l}\text { Farelo de trigo } \\
\text { Wheat meal }\end{array}$ & $\begin{array}{l}\text { Farelo de soja } \\
\text { Soybean meal }\end{array}$ & $\begin{array}{c}\text { Farinha de carne e ossos } \\
\text { Meat and bone meal }\end{array}$ & \\
\hline Total & $3.270,6$ & $3.357,1$ & $2.562,3$ & $3.217,7$ & $2.779,2$ & $3.037,4 \mathrm{~A}$ \\
\hline $\begin{array}{l}\text { Indicador } \\
\text { Marker }\end{array}$ & $3.208,3$ & $2.910,4$ & $2.133,5$ & $2.878,0$ & $2.894,6$ & $2.805,0 \mathrm{~B}$ \\
\hline
\end{tabular}

${ }^{1}$ Médias seguidas por letras distintas são diferentes $(P<0,05)$ pelo teste SNK.

${ }_{1}^{1}$ Means followed by distinct letters differ $(P<0.05)$ by SNK test.

Para o sorgo, o valor de EMA encontrado foi 15,13; 2,78 e $5,73 \%$ superior aos determinados com frangos, respectivamente, por Albino et al. (1981), Veloso et al. (1985) e Nagata et al. (2004). Para a EMAn, observou-se semelhança em relação aos valores apresentados por Coelho (1983) e superioridade de 3,94\%, em média, aos estimados para frangos (Veloso et al., 1985; Embrapa, 1991; NRC, 1994; Rostagno et al., 2000; Blas et al., 2003; Nagata et al., 2004).
A EMA do farelo de trigo foi, em média, 26,92\% maior que os determinados com frangos (Veloso et al., 1985; Albino et al., 1992; Borges et al., 2003) e 30,25\% superior à estimada com codornas (Silva et al., 2003; Torres, 2003). Do mesmo modo, o valor de EMAn foi $28,96 \%$ superior aos mencionados por Veloso et al. (1985), pela Embrapa (1991), por Albino et al. (1992), Rostagno et al. (2000) e Borges et al. (2003) e $24,69 \%$ maior que os descritos por Silva et al.(2003) e Torres (2003). Entretanto, foi 18,67\% menor que 
o reportado por Blas et al. (2003) e 8,27\% inferior ao obtido por Cilliers et al (1999), em avestruzes.

O valor de EMA do farelo de soja determinado pela técnica da coleta total foi 33,83\% maior que os determinados para frango (Coelho, 1983; Veloso et al., 1987; Albino et al., 1992; Rodrigues et al., 2002) e 25,78\% superior aos estimados com codornas (Silva et al., 2003; Torres, 2003). O valor de EMAn também foi $31,07 \%$ superior aos encontrados para frangos (Coelho, 1983; Veloso et al., 1987; Embrapa, 1991; Albino et al., 1992; Rostagno et al., 2000; Rodrigues et al., 2002; Blas et al., 2003; Zonta et al., 2004; Soares et al., 2005) e 27,48\% maior que os estimados com codornas (Silva et al., 2003; Torres, 2003), porém foi 13,62\% inferior ao determinado com avestruz (Cilliers, 1995).

Comparando os valores obtidos pela metodologia da coleta total, observa-se que a EMA e a EMAn da farinha de carne e ossos foram 37,23 e 35,19\% maiores, respectivamente, que os apresentados para frangos (Coelho, 1983; Veloso et al., 1987; Albino et al., 1992; Sartorelli, 1998; Brugalli et al., 1999) e 52,91 e 43,29\% superiores aos determinados com codornas (Torres, 2003). Entretanto, foram semelhantes aos encontrados por Soares et al. (2005). Essa variação entre os resultados pode ser atribuída às diferenças no teor de $\mathrm{PB}$ e MM nas diferentes farinhas de carne e ossos.

A média da EMA foi 4,48\% maior em relação à de EMAn dentro da metodologia de coleta total, corroborando a afirmação de Leeson \& Summers (2001) de que os valores de EM, quando corrigidos pelo balanço de nitrogênio, tendem a ser sempre menores, desde que as aves apresentem balanço de nitrogênio positivo, ou seja, estejam em crescimento, o que provavelmente explica os valores encontrados nesse trabalho. Do mesmo modo, observou-se superioridade de 5,46\% na média da EMA em relação à EMAn pela técnica do indicador.

As médias dos CMEB (Tabela 6) e CMEBn (Tabela 7) diferiram estatisticamente $(\mathrm{P}<0,05)$ entre as metodologias adotadas e os alimentos avaliados. Os valores encontrados pela técnica de coleta total foram 19,77 e 20,07\% (CMEB e CMEBn, respectivamente) maiores que os determinados com o uso do indicador. Entre os alimentos avaliados, notam-se maiores coeficientes para a farinha de carne e ossos, milho e o sorgo, que não diferiram $(\mathrm{P}>0,05)$ entre si, porém ambos foram maiores $(\mathrm{P}<0,05)$ quando comparados aos coeficientes do farelo de trigo. Essa diferença pode ser atribuída à maior porcentagem de FDN e FDA no trigo (em relação ao milho e ao sorgo) e à menor eficiência de metabolização da EB, visto que essas frações da FB, normalmente, apresentam baixa digestibilidade.

Segundo Lima (2001), o trigo contém inibidores de $\alpha$-amilase ainda não totalmente identificados, mas sabe-se que são proteínas encontradas principalmente no amido. As pentosanas solúveis do trigo apresentam efeito negativo sobre a digestibilidade do amido, da proteína e dos lipídeos nas dietas de aves (Choct et al., 1992) e, conseqüentemente, também afetam a metabolização da energia do trigo, o que explicaria os mais baixos coeficientes de metabolização da EB neste alimento.

Os CMEB e CMEBn do milho determinados pela técnica de coleta total foram semelhantes aos valores encontrados por Albino et al. (1981, 1992) e Rodrigues et al. (2001), entretanto o CMEBn foi menor que os encontrados por Coelho (1983), pela Embrapa (1991), por Rostagno et al. (2000) e Torres (2003) e superior ao CMEB determinado por Torres (2003) e D'Agostini et al. (2004).

No sorgo, o CMEB foi superior aos valores encontrados para frangos (Albino et al., 1981; Coelho, 1983; Nagata et al., 2004), do mesmo modo que o CMEBn foi maior que os obtidos por Albino et al.(1981), Coelho (1983), pela Embrapa (1991), por Rostagno et al. (2000) e Nagata et al. (2004).

O coeficiente de metabolização da energia bruta do farelo de trigo foi maior que os estimados em frangos (Albino et al., 1992; Borges et al., 2003), porém inferior ao

Tabela 6 - Coeficientes de metabolização aparente (CMEB) dos alimentos, determinados pela técnica de coleta total de excretas e com o uso de indicador

Table 6 - Values of the coefficients of apparent metabolization (CAMGE) of feedstuffs determined by the technique of total feces collection and use of marker

\begin{tabular}{|c|c|c|c|c|c|c|}
\hline \multirow[t]{2}{*}{$\begin{array}{l}\text { Técnica } \\
\text { Technique }\end{array}$} & \multicolumn{5}{|c|}{$\begin{array}{l}\text { CMEB do alimento } \\
\text { CAMGE of feedstuff }\end{array}$} & \multirow[t]{2}{*}{$\begin{array}{c}\text { Média }^{1} \\
\text { Mean }\end{array}$} \\
\hline & $\begin{array}{l}\text { Milho } \\
\text { Corn }\end{array}$ & $\begin{array}{c}\text { Sorgo } \\
\text { Sorghum }\end{array}$ & $\begin{array}{c}\text { Farelo de trigo } \\
\text { Wheat meal }\end{array}$ & $\begin{array}{l}\text { Farelo de soja } \\
\text { Soybean meal }\end{array}$ & $\begin{array}{c}\text { Farinha de carne e ossos } \\
\text { Meat and bone meal }\end{array}$ & \\
\hline Total & 82,40 & 85,83 & 70,11 & 82,38 & 96,65 & $83,48 \mathrm{~A}$ \\
\hline $\begin{array}{l}\text { Indicador } \\
\text { Marker }\end{array}$ & 71,69 & 68,27 & 49,97 & 68,25 & 76,70 & $66,98 \mathrm{~B}$ \\
\hline $\begin{array}{l}\text { Média }^{1} \\
\text { Average }^{2}\end{array}$ & $77,04 \mathrm{ab}$ & $77,05 \mathrm{ab}$ & $60,04 \mathrm{c}$ & $75,31 \mathrm{~b}$ & $86,68 \mathrm{a}$ & $\mathrm{CV}(\%)=14,77$ \\
\hline
\end{tabular}

\footnotetext{
${ }^{1}$ Médias seguidas por letras distintas são diferentes $(P<0,05)$ pelo teste SNK.
}

1 Means followed by distinct letters differ $(P<0.05)$ by SNK test. 
Tabela 7 - Coeficientes de metabolização aparente corrigida (CMEBn) dos alimentos, determinados pela técnica de coleta total de excretas e com o uso de indicador

Table 7 - Values of the coefficients of apparent corrected metabolization (CAMGEa) of feedstuffs determined by the technique of total feces collection and use of marker

\begin{tabular}{|c|c|c|c|c|c|c|}
\hline $\begin{array}{l}\text { Técnica } \\
\text { Technique }\end{array}$ & $\begin{array}{l}\text { Milho } \\
\text { Corn }\end{array}$ & $\begin{array}{l}\text { Sorgo } \\
\text { Sorghum }\end{array}$ & $\begin{array}{l}\text { Farelo de trigo } \\
\text { Wheat meal }\end{array}$ & $\begin{array}{l}\text { Farelo de soja } \\
\text { Soybean meal }\end{array}$ & $\begin{array}{c}\text { Farinha de carne e ossos } \\
\text { Meat and bone meal }\end{array}$ & $\begin{array}{c}\text { Média }{ }^{1} \\
\text { Mean }\end{array}$ \\
\hline Total & 82,20 & 84,30 & 67,26 & 76,09 & 88,26 & $79,62 \mathrm{~A}$ \\
\hline $\begin{array}{l}\text { Indicador } \\
\text { Marker }\end{array}$ & 71,66 & 66,91 & 47,46 & 62,05 & 70,10 & $63,64 \mathrm{~B}$ \\
\hline
\end{tabular}

${ }^{1}$ Médias seguidas por letras distintas são diferentes $(P<0,05)$ pelo teste SNK.

1 Means followed by distinct letters differ $(P<0.05)$ by SNK test.

determinado em codornas por Torres (2003). Valores inferiores foram obtidos por Albino et al. (1981), pela Embrapa (1991), por Albino et al. (1992), Rostagno et al. (2000) e Borges et al. (2003) e superiores ao descrito por Torres (2003) para o CMEBn.

Os valores obtidos pela técnica de coleta total para o farelo de soja foram superiores aos encontrados na literatura para frangos (Coelho, 1983; Veloso et al., 1987; Albino, 1981; Rodrigues, 2002; Soares, 2004; Zonta, 2004) e codorna (Torres 2003). Os coeficientes calculados para a farinha de carne e ossos também foram superiores aos apresentados para frangos (Albino et al., 1981; Coelho, 1983; Veloso et al., 1987; Albino et al., 1992; Brugalli, 1998; Sartorelli, 1998; Soares et al., 2005) e codornas (Torres, 2003).

Considerando os resultados obtidos, pode-se inferir que a metabolização da energia e a digestibilidade dos nutrientes são diferentes entre espécies (frango, codorna e avestruz) e variam conforme o alimento utilizado, principalmente quando os alimentos contêm altos teores de FDN e FDA, como o farelo de trigo e o farelo de soja. Torna-se evidente, portanto, que o uso de valores de EMAn normalmente utilizados para formulação de dietas para frangos subestima a utilização desta energia pelas emas.

\section{Conclusões}

A técnica de uso do indicador óxido de cromo mostrouse inadequada para determinar os CDAMS, CDAMO, EMA, EMAn, CMEB e CMEBn dos alimentos para emas, por apresentar valores inferiores aos obtidos pela técnica de coleta total de excretas.

Novos ensaios devem ser conduzidos para determinação das técnicas mais adequadas para estimativa dos valores energéticos e dos coeficientes de digestibilidade.

\section{Literatura Citada}

ALBINO, L.F.T.; ROSTAGNO, H.S.; FONSECA, J.B. et al. Tabela de composição de alimentos concentrados - Valores de composição química e de energia determinados com aves em diferentes idades. Revista da Sociedade Brasileira de Zootecnia, v.10, n.1, p.133-146, 1981.

ALBINO, L.F.T.; ROSTAGNO, H.S.; TAFURI, M.L. Determinação dos valores de energia metabolizável aparente e verdadeira de alguns alimentos para aves, usando diferentes métodos. Revista da Sociedade Brasileira de Zootecnia, v.21, n.6, p.1047-1058, 1992.

BLAS, C.; MATEOS, G.G.; REBOLlaR, P.G. Tablas FEDNA de composición y valor nutritivo de alimentos para la formulación de piensos compuestos. 2.ed. Madrid: Fundación Española para el Desarrollo de la Nutrición Animal, 2003. 423p.

BORGES, F.M.O.; ROSTAGNO, H.S.; SAAD, C.E.P. et al. Comparação de métodos de avaliação dos valores energéticos do grão de trigo e seus subprodutos para frangos. Arquivo Brasileiro de Medicina Veterinária e Zootecnia, v.55, n.6, p. 710-721, 2003.

BRUGalli, I.; ALBINO, L.F.T.; SILVA, D.J. et al. Efeito do tamanho de partículas e do nível de substituição nos valores energéticos da farinha de carne e ossos para pintos de corte. Revista Brasileira de Zootecnia, v.28, n.4, p.753-757, 1999

CHOCT, M.; ANNISON, G.; TRIMBLE, R. Soluble wheat pentosanas exhibit different anti-nutritive activies in intact and cecectomized broiler chickens. Journal Nutrition, v.122, p.2457-2465, 1992.

CILlLIERS, S.C.; HAYES, J.P.; MARITZ, J.S. et al. True and apparent metabolizable energy values of Lucerne and yellow maize in adult roosters and mature ostriches (Struthio camelus). Animal Production, v.59, p.309-313, 1994.

CILlliers, S.C.; HAYES, J.P.;CHWALIBOG, A. et al. A comparative study between mature ostriches (Struthio camelus) and adult cockerels with respect to true and apparent metabolisable energy values for maize, barley, oats and triticale. British Poultry Science, v.38, p.96-100, 1997.

CILLIERS, S.C.; SALES, J.; HAYES, J.P. et al. Comparison of metabolisable energy values of different foodstuffs determined in ostriches and poultry. British Poultry Science, v.40, p.491-494, 1999.

COELHO, M.G.R. Valores energéticos e de triptofano metabolizável de alimentos para aves, utilizando duas metodologias. Viçosa, MG: Universidade Federal de Viçosa, 1983. 77p. Dissertação (Mestrado em Zootecnia) - Universidade Federal de Viçosa, 1983. 
D’AGOSTINI, P.; GOMES, P.C.; ALBINO, L.F.T. et al. Valores de composição química e energética de alguns alimentos para aves. Revista Brasileira de Zootecnia, v.33, n.1, p. $128-134,2004$

EMPRESA BRASILEIRA DE PESQUISA E AGROPECUÁRIA EMBRAPA. Centro Nacional de Pesquisa de Suínos e Aves. Tabelas de composição química e valores energéticos de alimentos para suínos e aves. 3.ed. Concórdia: EMBRAPA/ CNPSA, 1991. 97p. (Documentos, 19)

LEESON, S.; SUMMERS, J.D. Scott's nutrition of the chicken. 4.ed. Guelph: University Books, 2001. 591p.

LIMA, G.J.M.M. Ingredientes energéticos: trigo e triticale na alimentação animal. In: SIMPÓSIO SOBRE INGREDIENTES NA ALIMENTAÇÃO ANIMAL, 2001, Campinas. Anais... Campinas: Colégio Brasileiro de Nutrição Animal, 2001. p.33-76.

MATTERSON, L.D.; POTTER, L.M.; STUTZ, M.W. et al. The metabolizable energy of feed ingredients for chickens. University of Connecticut Storrs. Agricultural Experiment Station Research Report, v.11, 11p, 1965

NAGATA, A.K.; RODRIGUES, P.B.; FREITAS, R.T.F. et al. Energia metabolizável de alguns alimentos energéticos para frangos de corte determinada por ensaios metabólicos e por equações de predição. Ciência e Agrotecnologia, v.28, n.3, p.674-683, 2004 .

NATIONAL RESEARCH COUNCIL - NRC. Nutrient requirements of poultry. 9.ed. Washington, D.C.: National Academy of Sciences, 1994. $155 \mathrm{p}$

PIMENTEL GOMES, F. Curso de estatística experimental. 1.ed. Piracicaba: Digaspari, 2000. 477p.

RODRIGUES, P.B.; ROSTAGNO, H.S.; ALBINO, L.F.T. et al. Valores energéticos do milheto, do milho e subprodutos do milho, determinados com frangos de corte e galos adultos. Revista Brasileira de Zootecnia, v.30, n.6, p.1767-1778, 2001.

RODRIGUES, P.B.; ROSTAGNO, H.S.; ALBINO, L.F.T. et al. Valores energéticos da soja e subprodutos da soja, determinados com frangos de corte e galos adultos. Revista Brasileira de Zootecnia, v.31, n.4, p.1771-1782, 2002.

ROSTAGNO, H.S.; ALBINO, L.F.T.; DONZELE, J.L. et al. Composição de alimentos e exigências nutricionais de aves e suínos (Tabelas Brasileiras). Viçosa, MG: Universidade Federal de Viçosa, 2000. 141p.

SANCHEZ, M.E.; LOUVANDINI, H.; NOGUEIRA, M. et al. Efeito de níveis de cálcio da dieta de filhotes de ema (Rhea americana, Linnaeus, 1758), na prevenção do entortamento de pernas, em cativeiro. Revista Brasileira de Zootecnia, v.27, n.6, p.86-90, 1998.

SARACURA, V.F. Crescimento inicial e entortamento de pernas de emas (Rhea maericana), em cativeiro, sob três níveis de energia na ração. Piracicaba: Escola Superior de Agricultura "Luiz de Queiroz", 1993. 108p. Dissertação (Mestrado em Ciência Animal e Pastagem) - Escola Superior de Agricultura "Luiz de Queiroz", 1993.
SARTORELLI, S.A.; BERTECHINI, A.G.; FASSANI, E.J. et al. Nutritional and microbiological evaluation of meat and bone meal produced in the State of Minas Gerais. Brazilian Journal of Poultry Science, v.5, n.1, p. 51-60, 2003.

STATISTICAL ANALYSIS SYSTEM - SAS. System for Microsoft Windows. Release 6.12, Cary: 1996. (CD-ROM).

SILVA, D.J.; QUEIROZ, A.C. Análise de alimentos: métodos químicos e biológicos. 3.ed. Viçosa, MG: Universidade Federal de Viçosa, 2002. 235p.

SILVA, J.B.G. Rheacultura criação de emas: manual prático nutrição, reprodução, manejo e enfermidades. Guaíba: Agropecuária, 2001. $144 \mathrm{p}$.

SILVA, J.H.V.; SILVA, M.B.; SILVA, E.L. et al. Energia metabolizável de ingredientes determinada com codornas japonesas (Coturnix coturnix japonica). Revista Brasileira de Zootecnia, v.32, n.6, p.1912-1918, 2003. (supl. 2).

SOARES, K.R.; FASSANI, E.J.; GERALDO, A. et al. Valores de energia metabolizável de alimentos para pintos de corte na fase préinicial. Ciência e Agrotecnologia, v.29, n.1, p.40-54, 2005.

SWART, D.; MACKIE, R. I.; HAYES, J. P. Influence of live mass, rate of passage and size of digestion on energy metabolim and fibre digestion in the ostrich (Struthio camelus var. domesticus). South African Journal of Animal Science, v. 23, n.5/6, p.119-126, 1993.

TORRES, A.F. Valor energético de sete alimentos e influência do diâmetro geométrico médio do milho para codorna francesa. Brasília: Universidade de Brasília, 2003. 50p. Dissertação (Mestrado em Ciências Agrárias) - Universidade de Brasília, 2003.

VELOSO, J.A.F.; SOUZA, T.C.R.; GOMES, A.V.C. et al. Composição química, matéria seca aparentemente metabolizável e conteúdo energético de alimentos energéticos para frangos de corte em diferentes idades. Arquivo Brasileiro de Medicina Veterinária e Zootecnia, v.37, n.2, p.157-167, 1985.

VELOSO, J.A.F.; GOMES, A.V.C.; SOUZA, T.C.R. et al Composição química e valores energéticos de alguns alimentos protéicos para frangos de corte em crescimento. Arquivo Brasileiro de Medicina Veterinária e Zootecnia, v.39 n.1, p.25-36, 1987.

ZONTA, M.C.M.; RODRIGUES, P.B.; FREITAS, R.T.F. et al Energia metabolizável de ingredientes protéicos determinada pelo método de coleta total e por equação de predição. Ciência e Agrotecnologia, v.28, n.6, p.1400-1407, 2004 